\title{
ANÁLISE DA PREVALÊNCIA DE DOENÇAS CRÔNICO-DEGENERATIVAS DE MORADORES DA CIDADE DE SANTO ANASTÁCIO - SP ATENDIDOS PELO PROJETO DE EXTENSÃO “MUTIRÃO DA SAÚDE”.
}

\author{
Alan José Barbosa Magalhães ${ }^{1}$, Tiarles Francisco do Nascimento ${ }^{2}$, Ana Paula Chesini Ribeiro ${ }^{2}$, Tatiana Emy \\ Koike ${ }^{1}$, Guilherme Akio Tamura Ozaki ${ }^{1}$, Thiago Alves Garcia ${ }^{1}$, Ariovaldo de Souza Ribeiro ${ }^{2}$, Marcelo José \\ Alves $^{2}$, Everton Alex Carvalho Zanuto ${ }^{2}$, Carlos Augusto de Carvalho Filho ${ }^{2}$, Marcelo Crepaldi Leitão ${ }^{2}$, Robson \\ Chacon Castoldi ${ }^{2,3}$, Regina Celi Trindade Camargo ${ }^{1}$, José Carlos Silva Camargo Filho ${ }^{1}$. \\ ${ }^{1}$ Universidade Estadual Paulista - UNESP, Presidente Prudente, SP, ${ }^{2}$ Universidade do Oeste Paulista - \\ UNOESTE, Curso de Educação Física, Presidente Prudente, SP, ${ }^{3}$ Universidade Estadual de Campinas - \\ UNICAMP.
}

\section{RESUMO}

O objetivo do estudo foi identificar a prevalência de doenças crônico-degenerativas (DCD) dos habitantes da cidade de Santo Anastácio-SP, atendidos pelo projeto "Mutirão da Saúde". Este estudo analisou 94 indivíduos, homens e mulheres, por meio do "Standard Health Questionnaire" (SHQ). A hipertensão arterial foi a DCD de maior prevalência em homens, enquanto o número de casos de doenças osteomusculares foi verificado em maior quantidade em mulheres. Em relação à idade, indivíduos acima de 40 anos apresentaram maior prevalência de todas as doenças. Ao ser realizada a estratificação por sexo/idade, mulheres acima de 40 anos apresentam maior prevalência de hipertensão arterial, doenças metabólicas e osteomusculares. Todos os achados nas estratificações citadas não apresentam relação direta dos participantes com sua hereditariedade. Conclui-se que há maior prevalência de hipertensão arterial em homens e doenças osteomusculares em mulheres. Além disso, foi observada maior prevalência de hipertensão arterial, doenças cardiovasculares e osteomusculares em homens e mulheres acima de 40 anos de idade.

Palavras-chave: doença crônica, hipertensão, doenças cardiovasculares.

\section{ANALYSIS OF THE PREVALENCE OF CHRONIC DISEASES OF SANTO ANASTÁCIO-SP'S RESIDENTS ATTENDED BY THE EXTENSION PROJECT “MUTIRÃO DA SAÚDE” (HEALTH’S MOVEMENT).}

\begin{abstract}
The aim of the study was to identify the prevalence of chronic-degenerative diseases (CDD) of the Santo Anastácio's population, attended by the project "Mutirão da Saúde" (Health's Movement). This study analyzed 94 subjects, men and women, through the "Standard Health Questionnaire" (SHQ). The arterial hypertension was the CDD of higher prevalence in men, while the number of musculoskeletal diseases cases was verified in greater quantity in women. In relation to age, individuals over 40 years old had a higher prevalence all diseases. When stratifying by sex/age, women over 40 years old have a higher prevalence of hypertension, metabolic and musculoskeletal diseases. All findings in the mentioned stratifications have no direct relationship of the participants with their heredity. We conclude that there is a higher prevalence of hypertension in men and musculoskeletal diseases in women. Besides, it was observed higher prevalence of hypertension, cardiovascular and musculoskeletal diseases in men and women over 40 years old.
\end{abstract}

Keywords: chronic disease, hypertension, cardiovascular diseases.

\section{INTRODUÇÃO}

Devido ao avanço tecnológico, a expectativa de vida da população tem aumentado e com isso, o aumento das doenças crônico-degenerativas $(D C D)^{1-3}$. As DCD são uma das principais causas de morte no Brasil e no mundo e podem ocorrer devido ao aumento da obesidade e do consumo de alimentos de altos valores energéticos, ricos em gordura ${ }^{4}$.

Segundo Camargo ${ }^{5}$ em 1940 a expectativa de vida não chegava aos 50 anos de idade (45,5 anos) e em 2011 esse indicador subiu 
para 74,08 anos, ou seja, a estimativa de vida subiu 28,58 anos. De acordo com o Instituto Brasileiro de Geografia e Estatística (IBGE) a expectativa de vida em 2050 deve subir para 81,3 anos.

Além disso, observa-se que tanto em países desenvolvidos quanto naqueles em desenvolvimento, estas DCD ocupam as primeiras posições nas estatísticas de mortalidade mundiais. Projeções para o ano de 2020 revelam que a mortalidade por DCD representará $73 \%$ dos óbitos no mundo ${ }^{6}$.

No Brasil, as DCD são também a principal causa de mortalidade. No ano de 2009, responderam por $72,4 \%$ do total de óbitos. Dentre os grupos de DCD mais comuns estavam: doenças cardiovasculares, neoplasias, doenças respiratórias crônicas e diabetes, que responderam por $80,7 \%$ dos óbitos por $\mathrm{DCD}^{7}$.

Estudos epidemiológicos demonstram que a inatividade física aumenta substancialmente a prevalência relativa de doença arterial coronariana (45\%), infarto agudo do miocárdio (60\%), hipertensão arterial (30\%), câncer de cólon (41\%), câncer de mama (31\%), diabetes do tipo II (50\%) e osteoporose $(59 \%)^{8}$. Além disso, o excesso de peso é um dos principais fatores para o risco para DCD. Estima-se que no ano de 2008, aproximadamente 36 milhões de mortes no mundo tenham sido causadas por $\mathrm{DCD}^{9,10}$.

Além de contribuir para a elevada mortalidade da população, as DCD são responsáveis por cerca de $65,5 \%$ dos anos vividos com deficiências e incapacidades nos países em desenvolvimento ${ }^{11}$, com repercussões negativas sobre a qualidade de vida (QV) dos indivíduos ${ }^{9}$. Segundo Capilheira e Santos ${ }^{12}$ o trabalho dos profissionais da saúde podem ser uma das formas mais efetiva de intervenção na prevenção das DCD.

Em 2011, o Brasil elaborou um plano de ações estratégicas para o enfrentamento da DCD nos próximos 10 anos com a participação do governo ${ }^{13}$. Muitas condições crônicas estão ligadas à uma sociedade em envelhecimento, mas também às escolhas de estilo de vida, como o tabagismo, consumo de álcool, comportamento sexual, dieta inadequada e inatividade física, além da predisposição genética ${ }^{14}$.

Dessa maneira, estudos que abordem essa temática são de fundamental importância para o avanço da ciência relacionada à saúde pública, pois pesquisas que possam realizar o levantamento de casos de doenças crônicas, podem servir de alerta para que autoridades competentes tomem iniciativas a fim de combater o surgimento de $\mathrm{DCD}$ na população brasileira. Logo, o objetivo do estudo foi identificar a prevalência de DCD dos habitantes da cidade de Santo Anastácio-SP, atendidos pelo projeto de extensão "Mutirão da Saúde".

\section{METODOLOGIA}

Os participantes da pesquisa constituem amostra por conveniência, não probabilística, sendo convidados a participar voluntariamente do estudo. A coleta foi realizada junto ao projeto de extensão "Mutirão da Saúde", promovido nas ações de responsabilidade social da UNOESTE atendendo a sociedade. Todos os participantes receberam e assinaram o Termo de Consentimento Livre e Esclarecido. Este projeto foi aprovado pelo Comitê de Ética da Universidade do Oeste Paulista - UNOESTE (processo $n^{\circ}$ 2464/2015).

A presente pesquisa analisou um grupo de 94 indivíduos com idade mediana de 39,5 \pm 26,5 anos de idade, de ambos os sexos (38 homens, sendo $17 \mathrm{com}$ idade $\leq 40$ anos e $21 \mathrm{com}$ idade > 40 anos; 56 mulheres, sendo $32 \mathrm{com}$ idade $\leq 40$ anos e 24 com idade $>40$ anos), residentes na cidade de Santo Anastácio - SP.

Os indivíduos foram distribuídos nos seguintes grupos: sexo (homens e mulheres); idade ( $\leq 40$ anos e $>40$ anos); sexo e idade (homens $\leq 40$ anos, homens $>40$ anos, mulheres $\leq 40$ anos e mulheres $>40$ anos).

Standard Health Questionnaire for Washington State (SHQ)

A ocorrência de DCD foi investigada a partir de um questionário de morbidade referida baseado no "Standard Health Questionnaire for Washington State" ${ }^{\prime 15}$. Assim, foi possível verificar a prevalência de $D C D$ no indivíduo avaliado e em seus familiares (pai e mãe).

O questionário investiga a ocorrência de doenças em um período de até 10 anos. No presente estudo foram abordados quatro grandes grupos de doenças, sendo elas: hipertensão arterial, doenças metabólicas endócrinas, doenças cardiovasculares e doenças osteomusculares.

\section{Análise estatística}

Foram determinados os pontos de corte de acordo com as variáveis "idade" e "sexo". 
Após a obtenção dos dados, foi aplicado o teste Qui-Quadrado para verificar a frequência "ocorrência de doenças" de pacientes e familiares (pai e mãe). Para isso, foi admitido um erro de $5 \%$. Tal procedimento foi realizado com o software IBM SPSS $22^{\circledR}$ for Windows ${ }^{\circledR}$.

\section{RESULTADOS}

Verifica-se que a hipertensão arterial teve maior prevalência no sexo masculino $(63,16 \%)$, enquanto nas mulheres foi de $50,0 \%$. Já a relação com a hereditariedade, a ocorrência foi de $34,22 \%$ para hipertensão arterial no sexo masculino e $50,0 \%$ no sexo feminino (Tabela 1 ).
Além disso, a prevalência de doenças osteomusculares para o feminino foi de $57,15 \%$, enquanto que para o sexo masculino foi de $28,95 \%$. Em comparação com as demais doenças, a prevalência de doenças metabólicas e cardiovasculares se apresentou baixa e de forma semelhante para ambos os sexos, assim como em seus familiares.

A Tabela 2 apresenta os resultados do SHQ para indivíduos com idades $\leq 40$ anos e $>40$ anos.

Tabela 1. Associação do sexo com doenças crônico-degenerativas apresentadas no questionário SHQ.

\begin{tabular}{|c|c|c|c|c|c|c|c|c|}
\hline \multicolumn{9}{|c|}{ Hipertensão Arterial } \\
\hline \multirow[b]{2}{*}{ Sexo } & \multirow[b]{2}{*}{ Presença } & \multicolumn{2}{|c|}{ Participantes } & \multirow{4}{*}{$\begin{array}{c}x^{2} \\
0,291\end{array}$} & \multicolumn{4}{|c|}{ Familiares } \\
\hline & & Ausência & Total & & Presença & Ausência & Total & $x^{2}$ \\
\hline Homens & 63,16 & 36,84 & 100 & & 34,22 & 65,78 & 100 & 0,144 \\
\hline Mulheres & 50,00 & 50,00 & 100 & & 50,00 & 50,00 & 100 & \\
\hline \multicolumn{9}{|c|}{ Doenças Metabólicas } \\
\hline & \multicolumn{4}{|c|}{ Participantes } & \multicolumn{4}{|c|}{ Familiares } \\
\hline Sexo & Presença & Ausência & Total & $x^{2}$ & Presença & Ausência & a Total & $x^{2}$ \\
\hline Homens & 13,16 & 86,84 & 100 & 0,131 & 10,53 & 89,47 & 100 & 0,110 \\
\hline Mulheres & 26,79 & 73,21 & 100 & & 25,00 & 75,00 & 100 & \\
\hline \multicolumn{9}{|c|}{ Doenças Cardiovasculares } \\
\hline & \multicolumn{4}{|c|}{ Participantes } & \multicolumn{4}{|c|}{ Familiares } \\
\hline Sexo & Presença & Ausência & Total & $x^{2}$ & Presença & Ausência & Total & $x^{2}$ \\
\hline Homens & 15,79 & 84,21 & 100 & 1,000 & 15,79 & 84,21 & 100 & 0,537 \\
\hline Mulheres & 16,08 & 83,92 & 100 & & 10,72 & 89,28 & 100 & \\
\hline \multicolumn{9}{|c|}{ Doenças Osteomusculares } \\
\hline & \multicolumn{4}{|c|}{ Participantes } & \multicolumn{4}{|c|}{ Familiares } \\
\hline Sexo & Presença & Ausência & Total & $x^{2}$ & Presença & Ausência & Total & $x^{2}$ \\
\hline Homens & 28,95 & 71,05 & 100 & 0,008 & 10,53 & 89,47 & 100 & 0,110 \\
\hline Mulheres & 57,15 & 42,85 & 100 & & 25,00 & 75,00 & 100 & \\
\hline \multicolumn{9}{|c|}{ Soma das Doenças } \\
\hline & \multicolumn{4}{|c|}{ Participantes } & \multicolumn{4}{|c|}{ Familiares } \\
\hline Sexo & Presença & Ausência & Total & $x^{2}$ & Presença & Ausência & Total & $x^{2}$ \\
\hline Homens & 76,32 & 23,68 & 100 & 0,799 & 50,00 & 50,00 & 100 & 0,298 \\
\hline Mulheres & 76,79 & 23,21 & 100 & & 67,86 & 32,14 & 100 & \\
\hline
\end{tabular}

Legenda: $\mathrm{X}^{2}$ = Teste do Qui-Quadrado. Valores percentuais em cada uma das categorias (\%). 
Tabela 2. Associação de idade com doenças crônico-degenerativas apresentadas no questionário SHQ.

\begin{tabular}{|c|c|c|c|c|c|c|c|c|}
\hline \multicolumn{9}{|c|}{ Hipertensão Arterial } \\
\hline & \multicolumn{4}{|c|}{ Participantes } & \multicolumn{4}{|c|}{ Familiares } \\
\hline Idade & Presença & Ausência & Total & $x^{2}$ & Presença & Ausência & Total & $x^{2}$ \\
\hline$\leq 40$ & 40,82 & 59,18 & 100 & 0,004 & 42,86 & 57,14 & 100 & 1,000 \\
\hline$>40$ anos & 71,12 & 28,88 & 100 & & 44,45 & 55,55 & 100 & \\
\hline \multicolumn{9}{|c|}{ Doenças Metabólicas } \\
\hline & \multicolumn{4}{|c|}{ Participantes } & \multicolumn{4}{|c|}{ Familiares } \\
\hline Idade & Presença & Ausência & Total & $x^{2}$ & Presença & Ausência & Total & $x^{2}$ \\
\hline$\leq 40$ anos & 16,33 & 83,67 & 100 & 0,313 & 20,41 & 79,59 & 100 & 0,798 \\
\hline$>40$ anos & 27,00 & 73,00 & 100 & & 17,78 & 82,22 & 100 & \\
\hline \multicolumn{9}{|c|}{ Doenças Cardiovasculares } \\
\hline & \multicolumn{4}{|c|}{ Participantes } & \multicolumn{4}{|c|}{ Familiares } \\
\hline Idade & Presença & Ausência & Total & $x^{2}$ & Presença & Ausência & Total & $x^{2}$ \\
\hline$\leq 40$ anos & 8,17 & 91,83 & 100 & 0,047 & 8,17 & 91,83 & 100 & 0,220 \\
\hline$>40$ anos & 24,45 & 75,55 & 100 & & 17,78 & 82,22 & 100 & \\
\hline \multicolumn{9}{|c|}{ Doenças Osteomusculares } \\
\hline & \multicolumn{4}{|c|}{ Participantes } & \multicolumn{4}{|c|}{ Familiares } \\
\hline Idade & Presença & Ausência & Total & $x^{2}$ & Presença & Ausência & Total & $x^{2}$ \\
\hline$\leq 40$ anos & 34,70 & 65,30 & 100 & 0,058 & 18,37 & 81,63 & 100 & 1,000 \\
\hline$>40$ anos & 57,78 & 42,22 & 100 & & 20,00 & 80,00 & 100 & \\
\hline \multicolumn{9}{|c|}{ Soma das Doenças } \\
\hline & \multicolumn{4}{|c|}{ Participantes } & \multicolumn{4}{|c|}{ Familiares } \\
\hline Idade & Presença & Ausência & Total & $x^{2}$ & Presença & Ausência & Total & $x^{2}$ \\
\hline$\leq 40$ anos & 69,39 & 30,61 & 100 & 0,020 & 61,23 & 38,77 & 100 & 0,456 \\
\hline$>40$ anos & 84,45 & 15,55 & 100 & & 60,00 & 40,00 & 100 & \\
\hline
\end{tabular}

Legenda: $\mathrm{X}^{2}$ = Teste do Qui-Quadrado. Valores percentuais em cada uma das categorias (\%).

Verifica-se maior presença de todas as variáveis de patologias apresentadas em participantes com idade $>40$ anos, em relação a indivíduos com idade $\leq 40$ anos. Além disso, a geração anterior apresenta maior ausência das patologias abordadas no questionário, em ambas as faixas etárias apresentadas (Tabela 2).

A Tabela 3 apresenta os resultados do SHQ para indivíduos de ambos os sexos, com idades $\leq 40$ anos $\mathrm{e}>40$ anos.
Foi verificada a prevalência de doenças cardiovasculares em indivíduos de ambos os sexos, com idade $>40$ anos de idade.

Quanto à hereditariedade, apenas a hipertensão arterial, em mulheres com idade $>40$ anos, apresenta maior ocorrência. Todas as outras variáveis apresentam prevalência da hereditariedade, em menor índice, semelhante ao que foi encontrado nos participantes de ambos os sexos (Tabela 3). 
Tabela 3. Associação de idade com doenças crônico-degenerativas apresentadas no questionário SHQ.

\begin{tabular}{|c|c|c|c|c|c|c|c|c|}
\hline \multicolumn{9}{|c|}{ Hipertensão Arterial } \\
\hline & \multicolumn{4}{|c|}{ Indivíduos } & \multicolumn{4}{|c|}{ Familiares } \\
\hline Idade & Presença & Ausência & Total & $x^{2}$ & Presença & Ausência & Total & $x^{2}$ \\
\hline $\mathrm{H} \leq 40$ anos & 58,83 & 41,17 & 100 & & 47,06 & 52,94 & 100 & \\
\hline $\mathrm{H}>40$ anos & 66,67 & 33,33 & 100 & & 23,81 & 76,19 & 100 & \\
\hline $\mathrm{M} \leq 40$ anos & 31,25 & 68,75 & 100 & & 40,63 & 59,37 & 100 & \\
\hline $\mathrm{M}>40$ anos & 75,00 & 25,00 & 100 & 0,006 & 62,50 & 37,50 & 100 & 0,071 \\
\hline \multicolumn{9}{|c|}{ Doenças Metabólicas } \\
\hline & \multicolumn{4}{|c|}{ Indivíduos } & \multicolumn{4}{|c|}{ Familiares } \\
\hline Idade & Presença & Ausência & Total & $x^{2}$ & Presença & Ausência & Total & $x^{2}$ \\
\hline $\mathrm{H} \leq 40$ anos & 5,89 & 94,11 & 100 & & 11,77 & 88,23 & 100 & \\
\hline $\mathrm{H}>40$ anos & 19,05 & 80,95 & 100 & & 9,53 & 90,47 & 100 & \\
\hline$M \leq 40$ anos & 21,88 & 78,12 & 100 & & 25,00 & 75,00 & 100 & \\
\hline$M>40$ anos & 33,34 & 66,66 & 100 & 0,207 & 25,00 & 75,00 & 100 & 0,377 \\
\hline \multicolumn{9}{|c|}{ Doenças Cardiovasculares } \\
\hline & \multicolumn{4}{|c|}{ Indivíduos } & \multicolumn{4}{|c|}{ Familiares } \\
\hline Idade & Presença & Ausência & Total & $x^{2}$ & Presença & Ausência & Total & $x^{2}$ \\
\hline $\mathrm{H} \leq 40$ anos & 5,89 & 94,11 & 100 & & 11,77 & 88,23 & 100 & \\
\hline $\mathrm{H}>40$ anos & 23,81 & 76,19 & 100 & & 19,05 & 80,95 & 100 & \\
\hline $\mathrm{M} \leq 40$ anos & 9,38 & 90,62 & 100 & & 6,25 & 93,75 & 100 & \\
\hline $\mathrm{M}>40$ anos & 25,00 & 75,00 & 100 & 0,191 & 16,77 & 83,33 & 100 & 0,511 \\
\hline \multicolumn{9}{|c|}{ Doenças Osteomusculares } \\
\hline & \multicolumn{4}{|c|}{ Indivíduos } & \multicolumn{4}{|c|}{ Familiares } \\
\hline Idade & Presença & Ausência & Total & $x^{2}$ & Presença & Ausência & Total & $x^{2}$ \\
\hline $\mathrm{H} \leq 40$ anos & 11,77 & 88,23 & 100 & & 11,77 & 88,23 & 100 & \\
\hline $\mathrm{H}>40$ anos & 42,86 & 57,14 & 100 & & 9,53 & 90,47 & 100 & \\
\hline$M \leq 40$ anos & 46,88 & 53,12 & 100 & & 21,88 & 78,12 & 100 & \\
\hline $\mathrm{M}>40$ anos & 70,84 & 29,16 & 100 & 0,006 & 29,17 & 70,83 & 100 & 0,312 \\
\hline \multicolumn{9}{|c|}{ Soma das Doenças } \\
\hline & \multicolumn{4}{|c|}{ Indivíduos } & \multicolumn{4}{|c|}{ Familiares } \\
\hline Idade & Presença & Ausência & Total & $x^{2}$ & Presença & Ausência & Total & $x^{2}$ \\
\hline $\mathrm{H} \leq 40$ anos & 64,81 & 35,19 & 100 & & 58,83 & 41,17 & 100 & \\
\hline $\mathrm{H}>40$ anos & 85,72 & 14,28 & 100 & & 42,86 & 57,14 & 100 & \\
\hline$M \leq 40$ anos & 71,88 & 28,12 & 100 & & 62,50 & 37,50 & 100 & \\
\hline $\mathrm{M}>40$ anos & 83,34 & 16,66 & 100 & 0,237 & 75,00 & 25,00 & 100 & 0,149 \\
\hline
\end{tabular}

Legenda: $\mathrm{H}$ = homem; $\mathrm{M}=$ Mulher. $\mathrm{X}^{2}=$ Teste do Qui-Quadrado. Valores percentuais em cada uma das categorias (\%).

\section{DISCUSSÃO}

O presente estudo teve como objetivo principal observar a ocorrência de DCD em moradores da cidade de Santo Anastácio-SP, atendidos pelo projeto de extensão universitária "Mutirão da Saúde", pertencente à Universidade do Oeste Paulista - Unoeste. Foi observado que houve maior prevalência de hipertensão arterial nos homens e doenças osteomusculares nas mulheres. Além disso, foi verificada maior prevalência em indivíduos com idade $>40$ anos, independente do sexo.

Segundo Francisco et al. ${ }^{14}$ doenças crônicas vêm se destacando no mundo como uma das principais causas de mortalidade em todo mundo. Nesse sentido, tal fato se torna mais grave em decorrência da idade, pois tais patologias são desenvolvidas em longo prazo e consequentemente, apresentam maior prevalência com o envelhecimento.

No presente estudo, foram investigados 94 indivíduos, sendo 37 foram do sexo masculino e 57 do sexo feminino, e verificado que o primeiro grupo apresentou prevalência de $62 \%$ de hipertensão arterial, enquanto que o segundo apresentou prevalência de 49,1\%.

Tal achado corrobora com o estudo de Francisco et al. ${ }^{14}$, que diz que hipertensão arterial 
é considerada um dos mais importantes fatores de risco para doenças crônicas. Segundo Gualano e Tinucci ${ }^{7}, D C D$ são as principais causas de morte no Brasil, das quais doenças cardiovasculares respondem pelo maior número de óbitos no país.

$\mathrm{O}$ presente estudo também observou a prevalência de hipertensão arterial nos familiares (pai e mãe), e verificou que $35 \%$ no sexo masculino e no $49,1 \%$ no feminino, apresentam esta patologia. Tal achado contraria os resultados de Longo et al. ${ }^{15}$, que para os fatores de proteção/risco para DCD na população total de ambos os sexos, observou-se que as mulheres apresentam maiores prevalências de comportamentos considerados como "fatores de proteção", em relação aos homens.

O presente estudo também verificou que a ocorrência de doenças metabólicas nos homens foi de $13,5 \%$, enquanto nas mulheres foi de $26,3 \%$. Já para os pais, a prevalência das doenças metabólicas no sexo masculino foi de $32,4 \%$ e no sexo feminino de $40,4 \%$. Segundo o estudo de Mariano et al., ${ }^{17}$ os principais fatores para 0 desenvolvimento de doenças metabólicas são o aumento da circunferência abdominal e excesso de peso.

Para a Organização Mundial de Saúde $(\mathrm{OMS})^{17}$, a síndrome metabólica caracteriza-se por resistência à insulina, ou alteração no metabolismo da glicose no organismo. Nesse sentido, políticas de prevenção poderiam ser adotadas com a finalidade de se reduzir a ocorrência dessas doenças, como o incentivo à prática de atividades físicas e adoção de hábitos alimentares saudáveis.

Outra variável observada no presente estudo foi a prevalência de doenças cardiovasculares. Nesse caso, foi verificado que $16,2 \%$ da população masculina avaliada apresentam casos dessas doenças, enquanto $15,8 \%$ da população feminina reportou alguma patologia deste grupo.

Já quando avaliados os pais dos entrevistados, $16,2 \%$ dos homens apresentaram alguma patologia deste grupo, enquanto no sexo feminino, foi observada a ocorrência de 10,5\%. Os achados expostos no presente estudo demonstram que pode haver o risco no desenvolvimento de doenças em aproximadamente $25 \%$ desta população. Segundo Gus et al. ${ }^{19}$, doenças cardiovasculares, em especial a doença arterial coronariana, representam a quinta causa de óbito em todo o mundo.
Por último, o presente estudo verificou a prevalência de doenças osteomusculares. Foi observado que a prevalência de $27 \%$ para o sexo masculino e $56 \%$ para o sexo feminino. Já nos pais, a prevalência foi de $10,8 \%$ para indivíduos do sexo masculino e $24,6 \%$ para o sexo feminino.

Em um estudo feito por Azevedo et al. ${ }^{10}$ em 920 indivíduos, a prevalência de doenças osteomusculares foi de 4,2\%, porcentagem inferior à encontrada no presente estudo. Estudos demonstram que a osteoporose acomete principalmente mulheres no período pósmenopausa, com menor nível de escolaridade ${ }^{12,20}$. Tal fenômeno deve-se provavelmente à diminuição da produção hormonal, ocorrida normalmente após os 50 anos de idade.

Assim, o presente estudo colabora com a literatura ao investigar a ocorrência de DCD em moradores da cidade de Santo Anastácio. No entanto, o estudo abordou apenas uma determinada localidade e população, assim como a ocorrência de DCD em indivíduos atendidos pelo programa de extensão universitária "Mutirão da Saúde". Estudos futuros, realizados em outras localidades e que abordem o estilo de vida, além dos fatores comportamentais, podem vir a colaborar com a presente pesquisa.

\section{CONCLUSÃO}

Conclui-se que houve maior prevalência de hipertensão arterial em homens e doenças osteomusculares em mulheres, atendidos pelo programa de extensão universitária "Mutirão da Saúde" no município de Santo Anastácio - SP. Além disso, todas as patologias demonstraram maior ocorrência em indivíduos com idade $>40$ anos.

\section{CONFLITO DE INTERESSES}

Os autores declaram não haver qualquer potencial conflito de interesses que possa interferir na imparcialidade deste trabalho científico.

\section{REFERÊNCIAS}

1. Costa LC, Thuler LCS. Fatores associados ao risco para doenças não transmissíveis em adultos brasileiros: estudo transversal de base populacional. Rev Bras Estud Popul. 2012;29(1):13345. DOI: $\quad$ http://dx.doi.org/10.1590/S010230982012000100009

2. Duncan BB, Chor D, Aquino EML, Bensenor IM, Mill JG, Schmidt $\mathrm{MI}$ et al. Doenças crônicas não transmissíveis no Brasil: prioridade para 
enfrentamento e investigação. Rev Saúde Pública. 2012;46(1):126-34 DOI:

http://dx.doi.org/10.1590/S0034-

\section{7}

3. Vilhena E, Ribeiro JLP, Silva I, Pedro L, Meneses LF, Cardoso $\mathrm{H}$, et al. Fatores psicossociais preditivos de ajustamento à vida de pessoas com doenças crônicas. Psic Saúde Doenças. 2014;15(1):220-33. DOI: http://dx.doi.org/10.15309/14psd150118

4. Azevedo ECC, Dias FMRS, Diniz AS, Cabral PC. Consumo alimentar de risco e proteção para as doenças crônicas não transmissíveis e sua associação com a gordura corporal: um estudo com funcionários da área de saúde de uma universidade pública de Recife (PE), Brasil. Ciênc Saúde Coletiva. 2014;19(5):1613-22.

DOI:

http://dx.doi.org/10.1590/1413-

\subsection{3}

5. Camargo MCS. Estimativas de expectativa de vida com doenças crônicas de coluna no Brasil. Ciênc Saúde Coletiva. 2014;19(6):1803-11.

DOI:

http://dx.doi.org/10.1590/1413-

\subsection{3}

6. Campos MO, Neto JFR, Silveira MF, Neves DMR, Vilhena JM, Oliveira JF et al. Impacto dos fatores de risco para doenças crônicas não transmissíveis na qualidade de vida. Ciênc Saúde Coletiva. 2013;18(3):873-82.

DOI:

http://dx.doi.org/10.1590/S1413-

81232013000300033

7. Gualano B, Tinucci T. Sedentarismo, exercício físico e doenças crônicas. Rev Bras Educ Fís Esporte. 2011;25:37-43. DOI: http://dx.doi.org/10.1590/S1807$\underline{55092011000500005}$

8. Horta PM, Cardoso AH, Lopes ACS, Santos LC. Qualidade de vida entre mulheres com excesso de peso e doenças crônicas não transmissíveis. Rev Gaúcha Enferm. 2013;34(4):121-9. DOI: http://dx.doi.org/10.1590/S1983-

14472013000400016

9. Borges TT, Rombaldi AJ, Knuth AG, Hallal PC. Conhecimento sobre fatores de risco para doenças crônicas: estudo de base populacional. Cad Saúde Pública. 2009;25(7):1511-20. DOI: http://dx.doi.org/10.1590/S0102-

\section{X2009000700009}

10. Azevedo AL, Silva RA, Tomasi E, Quevedo LA. Doenças crônicas e qualidade de vida na atenção primária à saúde. Cad Saúde Pública. 2013;29(9): 1774-82. DOI: $\quad$ http://dx.doi.org/10.1590/0102311X00134812

11. Capilheira M, Santos IS. Doenças crônicas não transmissíveis: desempenho no cuidado médico em atenção primária à saúde no sul do Brasil. Cad Saúde Pública. 2011;27(6):1143-53.

DOI:

http://dx.doi.org/10.1590/S0102-

\section{X2011000600011}

12. Iser BPM, Yokota RTC, Bandeira de Sá NN, Moura I, Malta DC. Prevalência de fatores de risco e proteção para doenças crônicas nas capitais do Brasil principais resultados do Vigitel 2010. Ciênc. Saúde Coletiva. 2012;17(9):2243-56. DOI: http://dx.doi.org/10.1590/S1413-

81232012000900015

13. Veras RP. Estratégias para o enfrentamento das doenças crônicas: um modelo em que todos ganham. Rev Bras Geriatr Gerontol. 2011;14(4):77986. DOI: $\quad$ http://dx.doi.org/10.1590/S180998232011000400017

14. Francisco PMS, Barros MBA, Segri NJ, Alves MCGP, Cesar CLG, Malta DCM. Comparação de estimativas para o auto-relato de condições crônicas entre inquérito domiciliar e telefônico - Campinas (SP), Brasil. Rev Bras Epidemiol. 2011;14(1):5-15. DOI: http://dx.doi.org/10.1590/S1415790X2011000500002

15. Longo GZ, Neves J, Castro TG, Pedroso MRO, Matos IB. Prevalência e distribuição dos fatores de risco para doenças crônicas não transmissíveis entre adultos da cidade de Lages (SC), sul do Brasil, 2007. Rev Bras Epidemiol. 2011;14(4):698-708. DOI: http://dx.doi.org/10.1590/S1415-

\section{X2011000400016}

16. Santos CE, Schrank Y, Kupfer R. Análise crítica dos critérios da OMS, IDF e NCEP para síndrome metabólica em pacientes portadores de diabetes melitos tipo 1. Arq Bras Endocrinol Metab. 2009;53(9):1096-102.

DOI:

http://dx.doi.org/10.1590/S0004-

\section{6}

17. Mariano KGTS, Ferreira SGS, Amaral IC, Oliveira LC. Identificação de fatores de risco para o desenvolvimento de síndrome metabólica e doença cardiovascular em estudantes universitários. Cad Escola de Saúde. 2014;2:50-62.

18. Moura EC, Silva AS, Malta DC, Neto OLM. Fatores de risco e proteção para doenças crônicas: vigilância por meio de inquérito telefônico, VIGITEL, Brasil, 2007. Cad Saúde Pública. 2011;27(3):486-96. DOI: http://dx.doi.org/10.1590/S0102-

\section{X2011000300009}

19. Gus S, Fischmann A, Medina C. Prevalência dos Fatores de Risco da Doença Arterial Coronariana no Estado do Rio Grande do Sul. Arq Bras Cardiol. 2002; 78(5):478-83.

DOI: https://doi.org/10.1590/S0066-782X2002000500005 20. Malta DC, Silva AS, Oliveira PPV, Iser BPM, Bernal RTI, Sardinha LMV, et al. Resultados do monitoramento dos Fatores de risco e Proteção para Doenças Crônicas Não Transmissíveis nas capitais brasileiras por inquérito telefônico, 2008. Rev Bras Epidemiol. 2012;15(3):639-50. DOI: http://dx.doi.org/10.1590/S1415790X2012000300017 